\title{
Acetabular Paralabral Cyst as a Rare Cause of Obturator Neuropathy: A Case Report
}

\author{
Sang-Hyun Kim, MD, Hyun Seok, MD, Seung Yeol Lee, MD, Sung Won Park, MD \\ Department of Physical Medicine and Rehabilitation, Soonchunhyang University Bucheon Hospital, \\ Soonchunhyang University College of Medicine, Bucheon, Korea
}

\begin{abstract}
An acetabular paralabral cyst is a benign soft tissue cyst usually seen in association with a tear of the acetabular labrum. Acetabular paralabral cysts are often the cause of joint pain, but they rarely cause compression of the adjacent neurovascular structures. We present a case of a 63-year-old male patient who had paresis and atrophy of right hip adductor muscles. Right obturator neuropathy was confirmed through an electrodiagnostic study. In addition, magnetic resonance imaging showed a paralabral cyst in the right acetabulum which extended to the pelvic wall. The patient underwent conservative treatment without surgical procedure. The pain was decreased after 1 month of conservative therapy. The pain was decreased at the 1-month follow-up. Follow-up electromyography showed polyphasic motor unit potentials in adductor magnus and adductor longus muscles. Based on the experience of this case, an acetabular paralabral cyst should be considered as one of the rare causes of obturator neuropathy.
\end{abstract}

Keywords Acetabulum, Synovial cyst, Obturator nerve

\section{INTRODUCTION}

A synovial cyst is a lesion that is generally found in large joints, such as the shoulders, knees, and hips. If the synovial cyst is associated with a tear of the acetabular labrum, it is termed as an acetabular labral cyst or an acetabular paralabral cyst. Tear of the acetabular labrum can result in loss of congruity between the femoral head

Received May 9, 2013; Accepted September 2, 2013

Corresponding author: Sung Won Park

Department of Physical Medicine and Rehabilitation, Soonchunhyang University Bucheon Hospital, Soonchunhyang University College of Medicine, 170 Jomaru-ro, Wonmi-gu, Bucheon 420-767, Korea

Tel: +82-32-621-5057, Fax: +82-32-621-5662, E-mail: pswness@gmail. com

(c) This is an open-access article distributed under the terms of the Creative Commons Attribution Non-Commercial License (http://creativecommons. org/licenses/by-nc/3.0) which permits unrestricted noncommercial use, distribution, and reproduction in any medium, provided the original work is properly cited.

Copyright $\odot 2014$ by Korean Academy of Rehabilitation Medicine and the acetabulum, which may lead to elevated intraarticular pressure. The elevated intra-articular pressure can force synovial fluid through the labral tear into the acetabulum or into the soft tissue adjacent to the acetabulum, resulting in an acetabular paralabral cyst. An acetabular paralabral cyst commonly causes pain and instability of the hip joint. However, it rarely compresses the adjacent nerve depending on its location [1].

The causes of obturator neuropathy include total hip arthroplasty, pelvic or abdominal procedures, inappropriate lithotomy position, pelvic tumor infiltration, and entrapment neuropathy, which is caused by compression of the nerve by the adductor muscle in athletes. A case of obturator neuropathy caused by an acetabular paralabral cyst is very rare, and only one such case has been reported [2]. Yukata et al. [2] reported obturator neuropathy based on clinical symptoms and magnetic resonance imaging (MRI) findings. However, we experienced a case 
of an acetabular paralabral cyst in the hip and obturator neuropathy caused by the acetabular paralabral cyst that was diagnosed using electromyography and MRI. Hence, we report the case of an acetabular paralabral cyst with literature review.

\section{CASE REPORT}

A 63-year-old male patient visited our hospital due to pain extending from the right medial thigh to the knee for one month. The patient had a history of traumatic rib fracture, which was caused by falling of a machine weighing $100 \mathrm{~kg}$ on his left flank four months ago, but he had completely recovered by the time of this visit. The patient was diagnosed with diabetes 20 years ago and had received drug treatment. He did not have any other significant diseases. Physical examination during this visit showed right femoral muscle atrophy and manual muscle

Table 1. Findings of the nerve conduction study

\begin{tabular}{|c|c|c|c|c|c|}
\hline Nerve & Stimulation & Recording & Latency (ms) & Amplitude $^{\text {a) }}$ & $\mathrm{CV}(\mathrm{m} / \mathrm{s})$ \\
\hline \multicolumn{6}{|l|}{ Motor } \\
\hline Rt. Tibial & Ankle & $\mathrm{AH}$ & 3.83 & 13.6 & 47.5 \\
\hline Lt. Tibial & Ankle & $\mathrm{AH}$ & 4.11 & 12.1 & 52.9 \\
\hline Rt. Peroneal & Ankle & EDB & 2.14 & 10.1 & 69.2 \\
\hline Lt. Peroneal & Ankle & EDB & 2.61 & 11.3 & 59.2 \\
\hline Rt. Obturator & Groin area ${ }^{\text {b) }}$ & $\mathrm{AM}$ & 2.30 & $1.3^{\mathrm{c})}$ & - \\
\hline Lt. Obturator & Groin area ${ }^{\text {b) }}$ & $\mathrm{AM}$ & 2.25 & 4.2 & - \\
\hline \multicolumn{6}{|l|}{ Sensory } \\
\hline Rt. Superficial peroneal & Lateral leg & foot & 2.20 & 10.7 & 54.5 \\
\hline Lt. Superficial peroneal & Lateral leg & foot & 2.50 & 10.4 & 52.0 \\
\hline Rt. Sural & Calf & Lateral malleolus & 1.98 & 10.6 & 45.5 \\
\hline Lt. Sural & Calf & Lateral malleolus & 1.55 & 10.0 & 53.1 \\
\hline
\end{tabular}

$\mathrm{CV}$, conduction velocity; Rt., right; Lt., left; $\mathrm{AH}$, abductor hallucis; $\mathrm{EDB}$, extensor digitorum brevis; AM, adductor magnus; CMAP, compound motor action potential.

${ }^{\text {a) }}$ Amplitudes are measured in millivolt ( $\mathrm{mV}$, motor) and in microvolt ( $\mu \mathrm{V}$, sensory). ${ }^{\mathrm{b}}$ Stimulation was performed through the needle electrode ${ }^{\mathrm{c}}{ }^{\mathrm{CMAP}}$ amplitude of the right obturator nerve was decreased compared with that of the left obturator nerve.

Table 2. Findings of needle electromyography

\begin{tabular}{lllll}
\hline \multicolumn{1}{c}{ Muscle } & IA & ASA & MUAP & Recruitment \\
\hline Rt. Lumbar PSP (L4-S1) & Normal & None & & \\
\hline Rt. Vastus medialis & Normal & None & Normal & Full \\
\hline Rt. Rectus femoris & Normal & None & Normal & Full \\
Rt. Gluteus medius & Normal & None & Normal & Full \\
\hline Rt. Gluteus maximus & Normal & None & Normal & Full \\
\hline Rt. Semitendinosus & Normal & None & Normal & Full \\
\hline Rt. Biceps femoris (long) & Normal & None & Normal & Full \\
\hline Rt. Tibialis anterior & Normal & None & Normal & Full \\
\hline Rt. Peroneus longus & Normal & None & Normal & Full \\
Rt. Gastrocnemius & Normal & None & Normal & Full \\
\hline Rt. Adductor magnus & Increased & ++ & Normal & Reduced \\
\hline Rt. Adductor longus & Increased & ++ & Normal & Reduced \\
\hline
\end{tabular}

IA, insertional activity; ASA, abnormal spontaneous activity; MUAP, motor unit action potential; PSP, paraspinal muscle. 
test revealed grade-IV muscle strength in the right hip adductors. The patient complained of pain in the right hip during active range of motion and passive range of motion, and especially, more severe right hip pain during extension and internal rotation. The sensory examination, deep tendon reflex examination, and pathologic reflex tests did not reveal any abnormal findings.

To identify the cause of right femoral muscle atrophy and pain, an electrodiagnostic study was performed. According to the electromyography findings, the amplitude of the compound motor action potential (CMAP) was reduced in the right obturator nerve compared to the left obturator nerve (Table 1). Denervation potentials were observed in the adductor magnus and adductor longus muscles with discrete recruitment patterns (Table 2). On the basis of these findings, the diagnosis of right obturator neuropathy was made.

To identify the cause of obturator neuropathy, contrastenhanced pelvic MRI was performed. The MRI showed a multi-lobular cyst measuring $2.7 \times 2.8 \mathrm{~cm}$ in the anteriorinferior right acetabulum and the cyst extended to the right obturator muscle and the inner wall of lesser pelvis (Fig. 1). The T1- and T2-weighted images showed that the right obturator muscle had atrophied compared to the left obturator muscle and it showed increased signal intensity. Contrast-enhanced T1-weighted image showed
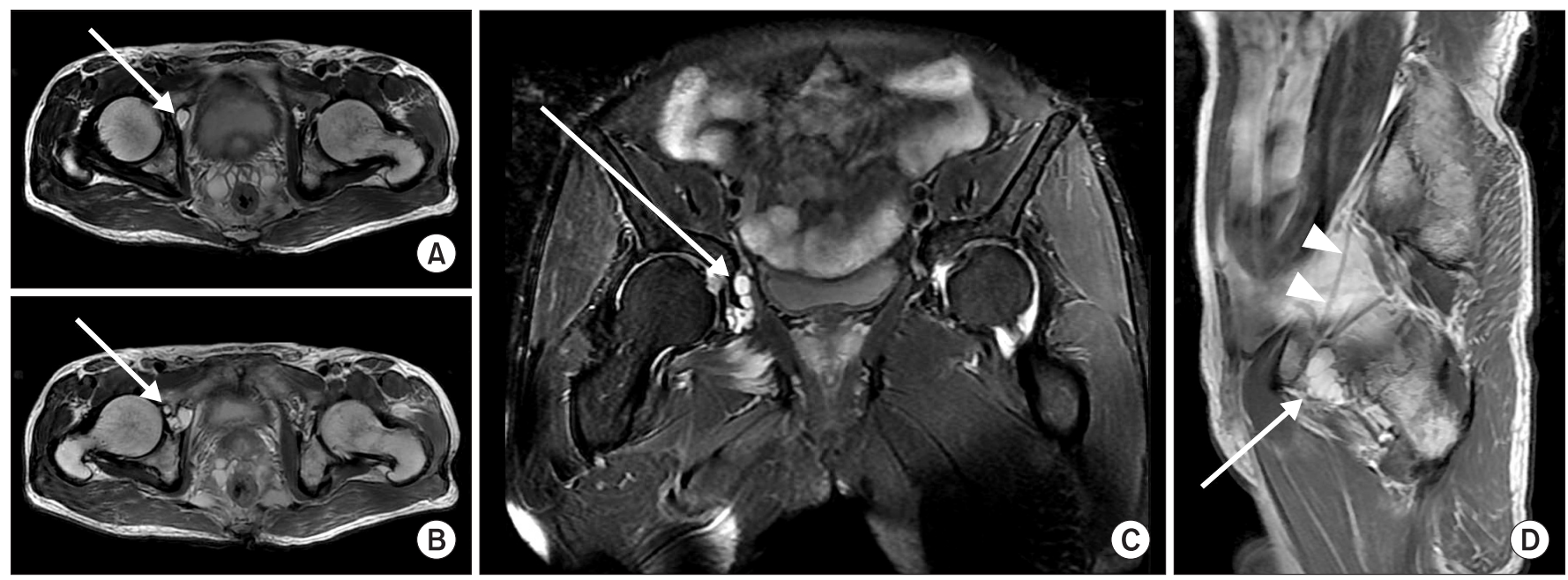

Fig. 1. T2-weighted magnetic resonance images of the pelvis. Axial image (A, B), coronal image (C), and parasagittal image (D) show an approximately $2.8 \mathrm{~cm}$ sized well-defined, lobulated, contoured, high signal intensity cystic lesion (arrow) in the anteroinferior aspect of the right acetabulum. This cystic lesion extended to the right obturator muscle and the lateral wall of lesser pelvis. (D) Parasagittal T2-weighted image demonstrates that the lobulated cystic lesion (arrow) extended along the course of the obturator nerve (arrowheads).
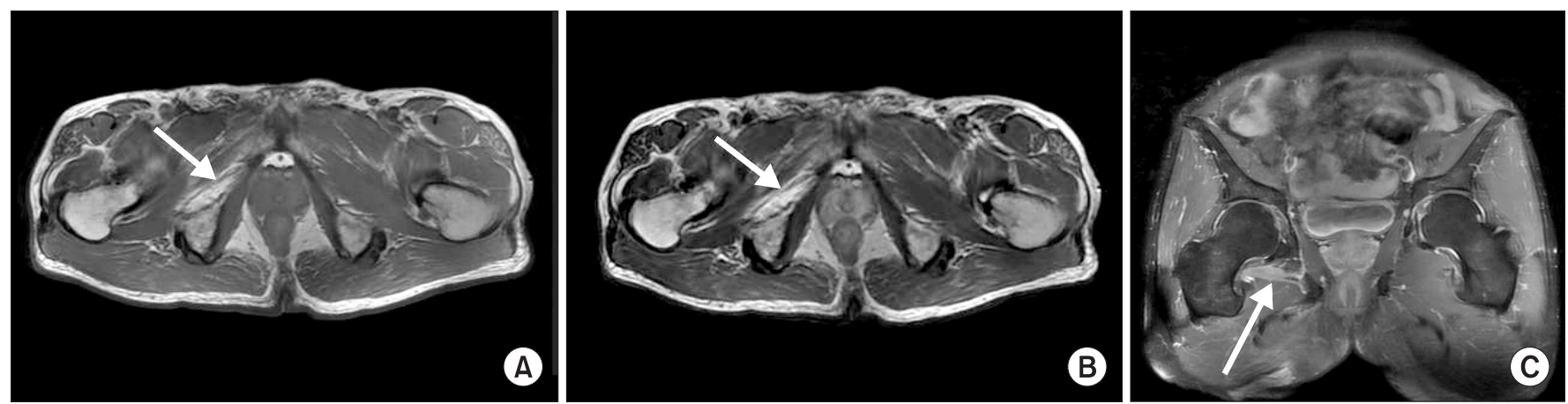

Fig. 2. Magnetic resonance imaging findings of the obturator muscle. Axial T1-weighted image (A), axial T2-weighted image (B), and gadolinium-enhanced coronal T1-weighted image (C) show high signal intensity in the right obturator muscle (arrow). These findings are suggestive of fatty atrophy. 
contrast enhancement in the right obturator muscle (Fig. 2). These findings suggest denervation damage with fatty atrophy. In the axial T2-weighted image, the obturator nerve was not observed due to cystic lesions in the right medial pelvic wall (Fig. 1A), and in the parasagittal T2-weighted image, an acetabular paralabral cyst was located under the pubic bone and it extended along the course of the obturator nerve (Fig. 1D).

We diagnosed obturator neuropathy caused by an acetabular paralabral cyst based on the reduction in the amplitude of CMAP of the obturator nerve, findings of denervation potentials in the adductor magnus and adductor longus on electromyography, and findings of denervation damage in the obturator muscle and an acetabular paralabral cyst that extended along the course of the obturator nerve to the pelvic wall on MRI.

Then, we initiated conservative therapy and followups to decide whether to perform surgical or nonsurgical treatment. Clinical symptoms, such as pain in the right thigh and knee, were relieved within a month after diagnosis, but right adductor muscle atrophy and decrease in the strength of the right hip adductor muscle to $4 / 5$ on manual muscle test persisted. On follow-up electrodiagnostic study, CMAP of the right obturator nerve was slightly improved in a nerve conduction study, but the amplitude of the CMAP of the right obturator nerve was still less than that of the CMAP of the left obturator nerve (Table 3). On needle electromyography, denervation potential and reduced recruitment pattern in the adductor

Table 3. Findings of follow-up obturator nerve conduction study

\begin{tabular}{lccc} 
Motor nerve & $\begin{array}{c}\text { Latency } \\
(\mathbf{m s})\end{array}$ & $\begin{array}{c}\text { Amplitude } \\
(\mathbf{m V})\end{array}$ & $\begin{array}{c}\text { CV } \\
(\mathbf{m} / \mathbf{s})\end{array}$ \\
\hline Rt. Obturator & 2.31 & $2.4^{\mathrm{a})}$ & - \\
\hline Lt. Obturator & 2.27 & 4.3 & - \\
\hline
\end{tabular}

$\mathrm{CV}$, conduction velocity; CMAP, compound motor action potential.

${ }^{\text {a) }}$ CMAP amplitude of the right obturator nerve was decreased compared with that of the left obturator nerve. magnus and adductor longus was maintained, but polyphasic motor unit action potentials were newly observed (Table 4).

The patient showed incomplete damage to the obturator nerve due to the acetabular paralabral cyst and the strength of the hip adductor muscles persistently decreased after conservative therapy; but pain in the right thigh and knee was improved. The patient consulted an orthopedic surgeon regarding the need for surgery, and it was decided to observe the progress without performing surgery because of the improvement in clinical symptoms.

\section{DISCUSSION}

The obturator nerve originates from the anterior division of the ventral rami of the second, third, and fourth lumbar spinal nerves. The obturator nerve descends through the psoas muscle and enters the thigh through the upper part of the obturator foramen along the inner wall of lesser pelvis. Then, the obturator nerve divides into anterior and posterior branches. The anterior branch innervates the adductor longus, gracilis, and adductor brevis and also gives off sensory fibers that innervate the skin and fascia of the medial aspect of the midthigh. The posterior branch innervates the external obturator muscle and runs between adductor brevis and adductor magnus muscles. Then, it splits into a motor branch that innervates adductor magnus and a sensory branch that descends to the knee joint [3].

The symptoms of obturator neuropathy include medial femoral paresthesia, pain, decreased muscle strength during hip adduction and internal rotation, and hip adductor muscle atrophy. It was reported that pain in the medial thigh and the groin was observed more frequently than hypesthesia and dysesthesia [3,4]. Pain due to obturator neuropathy may extend from the origin of the adductor muscle in the pubic bone to the medial thigh and knee, and extension or abduction of hips can induce or aggravate pain due to stretching of the obturator nerve [3].

Table 4. Findings of follow-up needle electromyography

\begin{tabular}{llccc}
\hline \multicolumn{1}{c}{ Muscle } & IA & ASA & MUAP & Recruitment \\
\hline Rt. Adductor magnus & Increased & ++ & Polyphasic & Reduced \\
Rt. Adductor longus & Increased & ++ & Polyphasic & Reduced \\
\hline
\end{tabular}

IA, insertional activity; ASA, abnormal spontaneous activity; MUAP, motor unit action potential. 
This patient had pain in the medial thigh and knee, and weak adductor muscles; but he did not suffer from paresthesia. Similarly, Yukata et al. [2] reported tenderness in the adductor muscles, but absence of paresthesia.

There are many variations in the sensory distribution of the obturator nerve. Bouaziz et al. [5] reported that the obturator nerve did not affect cutaneous sensation in $57 \%$ of the subjects, and this finding was determined by performing obturator nerve block. Therefore, in our case, it was considered that paresthesia in the medial midthigh was not due to variation in the sensory distribution of the obturator nerve.

Diagnosis of obturator neuropathy can be established by confirming adductor muscle atrophy through MRI. But, MRI cannot detect nerve disorders directly, and therefore, the diagnosis of obturator neuropathy should be confirmed by performing electromyography [3]. Not many nerve conduction studies of obturator nerves have been reported, and the method has not yet been established. In our case, we compared CMAP of the obturator nerves in both the sides by stimulating the nerves with a needle electrode and confirmed obturator neuropathy based on the reduction in amplitude of the CMAP in the right obturator nerve, and the abnormal spontaneous activity in the right adductor magnus and adductor longus on needle electromyography.

Acetabular paralabral cyst may be detected in patients who have developmental dysplasia of the hip, osteoarthritis, and hip joint trauma [1]. Degeneration and tear of the acetabular labrum can occur due to shear force imposed on the hip joint of the patients who have these lesions. Consequently, femoral head does not fit into the acetabulum, which causes an increase in the intraarticular pressure, and this elevated intra-articular pressure pushes out the soft tissue of the hip through the torn acetabulum, resulting in an acetabular paralabral cyst. Therefore, in most of the cases, an acetabular paralabral cyst is found in association with a tear of the acetabular labrum [6].

The link between the articular cavity and the acetabular cyst caused by a tear of the acetabular labrum is generally not diagnosed by conventional MRI, and it is diagnosed by magnetic resonance arthrography. However, Magee and Hinson [7], who investigated the relation between acetabular labral lesions and acetabular paralabral cysts, reported that all of the patients who had acetabular paralabral cysts on conventional MRI showed tears of the acetabular labrum on magnetic resonance arthrography and the presence of acetabular paralabral cysts was eventually an indirect finding to strongly suggest tears of the acetabular labrum. Magnetic resonance arthrography was not performed in our patient, and therefore, a tear of the acetabular labrum could not be confirmed; but, we presumed that there was a tear of the acetabular labrum because an acetabular paralabral cyst was detected on MRI.

The incidence and location of acetabular paralabral cysts have not been clearly defined [8]. Magerkurth et al. [9] evaluated the presence of paralabral cysts of the hip joint using magnetic resonance arthrography, and only 18 out of 704 patients were found to have acetabular paralabral cysts. In this study, the location of acetabular paralabral cysts was anterosuperior in $56 \%$ of patients, anterior in $22 \%$ of patients, posterosuperior in $17 \%$ of patients, and anteroinferior in only $6 \%$ of patients [9]. In our patient, the acetabular paralabral cyst was located in the anterior-inferior acetabulum, posterior to the pubic bone, and the lateral wall of lesser pelvis, and it extended along the course of the obturator nerve in the posterior pubic bone. Yukata et al. [2] also reported that the cyst extended from the anterior acetabulum to the lateral wall of lesser pelvis. Blood supply to the anterior acetabular labrum is less than that to the other sites, and hence, the anterior acetabular labrum may be vulnerable to injury. Also, it is considered that the labrum, articular capsule, and ligaments mainly provide stability to the anterior acetabulum and head of the femur rather than the bony portion [8]. We suggest that the soft tissue of the anterior acetabulum is more vulnerable to injury than the other portion.

According to Magerkurth et al. [9], 94\% of labral cysts were lobular and $78 \%$ of labral cysts extended to the outside of the acetabulum and showed superior extension. In our patient and in the case presented in the study by Yukata et al. [2], acetabular paralabral cysts were lobular and showed superior extension along the lateral wall of lesser pelvis. This may have occurred because the intraarticular fluid was gradually pushed into the cyst through the torn acetabular labrum due to an intermittent increase in intra-articular pressure in the hips while walking.

Acute obturator neuropathy tends to have good prognosis after conservative therapy. Sorenson et al. [4] reported that 14 out of 15 patients with acute obturator neuropa- 
thy showed good recovery and improvement in function. Our patient did not undergo surgery after considering the surgical risk, and he received continuous conservative therapy and was observed regularly. The pain in the right medial aspect of the thigh was gradually relieved within a month, and polyphasic motor unit action potentials in adductor magnus and adductor longus muscles were observed on follow-up needle electromyography. Yukata et al. [2] reported that the pain while walking was improved at one month after aspiration of acetabular paralabral cysts.

The hip joint is in close proximity to the anterior femoral nerve, the posterior sciatic nerve, and the medial obturator nerve. Therefore, there is a substantial probability that an acetabular paralabral cyst causes damage to the nerves that are located adjacent to the hip joint $[2,10]$. Acetabular paralabral cysts are generally not detected on plain radiography unlike fracture and dislocation of the hips. Therefore, if a patient with clinical manifestations of lower limb neuropathy complains of pain in the hips, the possibility of neuropathy due to hip disorders, such as acetabular paralabral cysts, should be considered.

\section{CONFLICT OF INTEREST}

No potential conflict of interest relevant to this article was reported.

\section{ACKNOWLEDGMENTS}

This research was supported by the Soonchunhyang University Research Fund.

\section{REFERENCES}

1. Steiner E, Steinbach LS, Schnarkowski P, Tirman PF, Genant HK. Ganglia and cysts around joints. Radiol Clin North Am 1996;34:395-425.

2. Yukata K, Arai K, Yoshizumi Y, Tamano K, Imada K, Nakaima N. Obturator neuropathy caused by an acetabular labral cyst: MRI findings. AJR Am J Roentgenol 2005;184(3 Suppl):S112-4.

3. Tipton JS. Obturator neuropathy. Curr Rev Musculoskelet Med 2008;1:234-7.

4. Sorenson EJ, Chen JJ, Daube JR. Obturator neuropathy: causes and outcome. Muscle Nerve 2002;25:6057.

5. Bouaziz H, Vial F, Jochum D, Macalou D, Heck M, Meuret P, et al. An evaluation of the cutaneous distribution after obturator nerve block. Anesth Analg 2002;94:445-9.

6. Schnarkowski P, Steinbach LS, Tirman PF, Peterfy CG, Genant HK. Magnetic resonance imaging of labral cysts of the hip. Skeletal Radiol 1996;25:733-7.

7. Magee T, Hinson G. Association of paralabral cysts with acetabular disorders. AJR Am J Roentgenol 2000;174:1381-4.

8. Lewis CL, Sahrmann SA. Acetabular labral tears. Phys Ther 2006;86:110-21.

9. Magerkurth O, Jacobson JA, Girish G, Brigido MK, Bedi A, Fessell D. Paralabral cysts in the hip joint: findings at MR arthrography. Skeletal Radiol 2012;41:1279-85.

10. Jones HG, Sarasin SM, Jones SA, Mullaney P. Acetabular paralabral cyst as a rare cause of sciatica: a case report. J Bone Joint Surg Am 2009;91:2696-9. 